\title{
THYROID, THYMUS, Etc.
}

Taillens.-Death from Hypertrophy of the Thymus Gland. "Revue Méd. de la Suisse Romande," June 20, 1901.

The author has seen two deaths in children due to hypertrophy of the thymus :

Case 1.-A boy, fourteen days old, born of healthy parents at full time, and in all respects healthy, was fed at half-past nine, put to bed at ten, where he soon fell asleep. The mother left him sleeping perfectly naturally, but on returning after about two and a half hours found him suffocating, cyanosed. After some hours he died asphyxiated. At the post-mortem examination all the organs were found healthy except the thymus gland, which was much enlarged, measuring 4 to 5 centimetres long, $7 \frac{1}{2}$ centimetres broad, and $1 \frac{1}{2}$ to 2 centimetres thick, and weighing 38 grammes-i.e., four times the normal weight. The trachea was flattened in its antero-posterior axis, at the junction of the cervical and thoracic portions, by the pressure of the enlarged thymus. The large vessels were not affected. The heart was in diastole, and the blood liquid.

Case 2.-A girl, two years and four months old; father had a goitre, mother healthy; six brothers and sisters healthy. In March, 1900, had diphtheria, but made a good recovery; frequent disorders of digestive tract, with diarrhœa, up till age of one year and a half, since then healthy. On February 14, 1901, commencing loss of appetite, irritability, slight fever at night, no cough, no respiratory difficulty. On February 16 she was left in charge of a girl, fifteen years old, who gave her a drink, and placed her in bed, lying on her left side. After about three hours, during which the attendant never left the room, the mother returned and found the child dead, lying in the exact position in which it had been laid.

At the post-mortem all the organs were found healthy-no signs of rickets-except the thymus, which was enormous. The enlargement, however, was such as to cause pressure, not on the trachea, which was quite normal, but on the roots of the large vessels, especially the pulmonary artery. The leart was arrested in systole.

After discussing shortly the physiology and pathology of the thymus, and rather fully the different views of the " mechanism of death" in hypertrophy of the thymus the author sums up as follows:

1. It cannot be doubted that in certain cases hypertrophy of the thymus can be a cause of death.

2. Death may be sudden, due to syncope, or more slow, due to asphyxia.

3. In the former case there can be no question of treatment, because death occurs so rapidly that there is no time to apply remedies. In the latter case-i.e., death from compression of trachea-medical treatment is useless, so also is intubation or tracheotomy. Only one case has been successfully treated by operation. (Case of Siegle, operated by Rehn. Tracheotomy, introduction of long tube down to bifurcation of trachea. This tube caused so much irritation it could not be tolerated, but on its removal the dyspnœa at once returned. Second operation: Extension of tracheotomy wound down to sternum, opening of anterior mediastinum, fixation of thymus by a few stitches to supra-sternal fascia.)

4. The importance of enlargement of the thymus may be great, specially in forensic medicine. The thymus may be the real cause of 
death in certain rare cases of so-called death from goitre; it may also be the cause of the difficulty of decannulation after certain tracheotomies.

5. The expression " thymic asthma" is not exact. It is better to speak of the rapid form of death as "cardiac or sudden death due to the thymus," and of the asphyxial form as "thymic tracheostenosis." Arthur J. Hutchison.

\section{NOSE, Etc.}

\section{Du Fougeray, Hamon (Le Mans).-The Rhino-pharyngeal Origin of} Goitre. "Le Progrès Médical," No. 21, May, 1901.

In an interesting article the author gives the results of five years' research ; during this time he treated over 200 cases of goitre in which naso-pharyngeal lesions were also present. Fifty-two of these cases were quite cured by suitable naso-pharyngeal treatment in periods varying from one to eight months. In 133 cases the goitre, being of old standing, fibrous or cystic, was considerably reduced in size. In 24 cases the swelling was only slightly reduced. Some goitres of over twenty years' duration were reduced 2 to 4 inches.

The author states that in all these cases a previous congestive state of the naso-pharynx had existed, which would react on the thyroid through the venous anastomosis, described by Bimar and Lapeyre (Transactions of the Academy of Science, Paris, 1897). He believes that a vascular lesion of the oro-pharynx produces goitre; a vascular lesion of the nose produces exophthalmus; and that mixed lesions, vascular and sympathetic, of the oro-pharynx produce exophthalmic goitre and cardiac mischief.

The treatment consisted in the use of a 10 per cent. menthol spray three times daily, and the use of a 50 per cent. solution of chromic acid as a caustic for enlarged turbinates or pharynx.

Anthony McCall.

Dunbar, Roy.-Case of Nasal Sarcoma. "Journal Amer. Med. Assoc.," August 10, 1901.

Record of a case of nasal sarcoma in which ligature of the external carotids was tried with the view of starving the nasal growth. Very little effect was, however, produced, as the patient died seven months after the first appearance of symptoms.

W. Milligan.

Holmes, C. R., and Garlich, H. S.-Accidents attending Adenoid Operations. "Laryngoscope," May, 1901.

In Dr. Holmes's case the patient, a female, aged eight, was undergoing an operation for removal of naso-pharyngeal adenoids under chloroform anæsthesia. When the curette had been introduced into the naso-pharynx, and just as pressure was being made, the instrument suddenly snapped. With great difficulty the fragment was drawn down into the oro-pharynx, and removed by means of a pair of forceps.

In Dr. Garlich's case the patient was being operated upon without an anæsthetic. The instrument suddenly snapped, and the broken fragment was swallowed. Suitable diet was prescribed, and the broken fragment, $\frac{1}{2}$ inch long and $\frac{1}{16}$ inch broad, was passed per rectum three days afterwards. 